\title{
Hybrid-State Dynamics of Dye Molecules and Surface Plasmon Polaritons under Ultrastrong Coupling Regime
}

\author{
Hai Wang, Hai-Yu Wang, * Qi-Dai Cheng, Huai-Liang Xu, Hong-Bo Sun,* \\ Fangcheng Huang, Waseem Raja, Andrea Toma, and Remo Proietti Zaccaria*
}

The achievement of an ultrastrong coupling regime between surface plasmon modes generated by gold conical pits arrays and excitons associated to squaraine dye is demonstrated. Numerical and experimental steady-state reflection measurements demonstrate a remarkable Rabi splitting of $860 \mathrm{meV}$, to date the largest reported value involving surface plasmon modes.

Furthermore, the dynamics of the hybrid states under the ultrastrong coupling regime is investigated by transient absorption spectroscopy. The results show that the upper bands are too short-lived to be detected, while the lower bands have a relatively shorter lifetime with respect to the bleaching recovery of pure squaraine dye. This result contradicts the behaviour of systems in strong coupling regimes, suggesting a different photophysics between strong and ultrastrong coupled systems.

\section{Introduction}

Among the many research fields which have found a new youth due to the advancements in nanotechnology, surely takes its place the study of the interactions between light and matter. ${ }^{[1,2]}$ These interactions can be classified into two different regimes based on

\footnotetext{
Dr. H. Wang, Prof. H.-Y. Wang, Prof. Q.-D. Chen, Prof. H.-L. Xu, Prof. H.-B. Sun

State Key Laboratory of Integrated Optoelectronics

College of Electronic Science and Engineering

Jilin University

Changchun 130012, China

E-mail: haiyu_wang@jlu.edu.cn; hbsun@jlu.edu.cn

Dr. A. Toma, Prof. R. Proietti Zaccaria

Istituto Italiano di Tecnologia

via Morego 30, 16163, Genova, Italy

E-mail: remo.proietti@iit.it; remo.proietti@nimte.ac.cn

Dr. W. Raja

Nanooptische Konzepte für die PV

Helmholtz-Zentrum Berlin

Hahn-Meitner-Platz 1, 14109, Berlin, Germany

F. Huang, Prof. R. Proietti Zaccaria

Cixi Institute of Biomedical Engineering

Ningbo Institute of Materials Technology and Engineering

Chinese Academy of Sciences

Ningbo 315201 , China

Prof. H.-B. Sun

State Key Lab of Precision Measurement Technology and Instruments

Department of Precision Instrument

Tsinghua University

Haidian, Beijing 100084, China
}

their strength: weak and strong coupling. In particular, the weak coupling regime describes the situation where the energy levels of the material remain essentially unaltered. The interaction with light only modifies the spontaneous emission rate, while the emission frequency is unchanged. ${ }^{[3,4]}$ On the other hand, the strong coupling regime describes a lightmatter interacting system where a reversible energy exchange between electromagnetic modes (light) and electronic levels (matter) will take place before any dissipation or decoherence process can occur. $^{[5-10]}$ Under this regime, the matter wavefunctions and electromagnetic modes are in a coherent superpositions, leading to two hybrid light-matter states with intriguing properties, capable of leading for example to thresholdless lasing, ${ }^{[11-14]}$ to the possibility to achieve Bose-Einstein condensation at high temperatures ${ }^{[15]}$ or for the realization of quantum entangled states. At resonance, the energy gap between the hybrid states is known as Rabi splitting. To date, various approaches including optical microcavity and surface plasmon polaritons (SPPs) have been used to demonstrate strong coupling with J-aggregates molecules, ${ }^{[16-20]}$ dye molecules ${ }^{[21-23]}$ and quantum dots. ${ }^{[24-26]}$

Recently, with the introduction of photochromic molecules and organic semiconductors with inhomogeneous broadened absorption spectra, the light-matter interaction entered a completely new regime, in which the Rabi splitting values are remarkably enhanced. In the so-called ultrastrong coupling regime, the Rabi splitting energy becomes an appreciable fraction of the exciton transition energy, which has been experimentally and theoretically observed in a variety of systems. ${ }^{[27-30]}$ A Rabisplitting of $700 \mathrm{meV}$ was firstly observed in a microcavity system by employing photochromic molecules. ${ }^{[27]}$ Since then, giant Rabi-splitting values exceeding $1 \mathrm{eV}$ were reached with organic semiconductors. ${ }^{[28,29]}$ Meanwhile, ultrastrong coupling was also achieved through the interaction of matter with SPPs. The observed Rabi splitting was comparable to the value obtained in the microcavity. Cacciola et al. ${ }^{[30]}$ reported a giant Rabi splitting of $780 \mathrm{meV}$ in nanoshells constituted by a core of organic molecules surrounded by a nanometric shell. Indeed, plasmonic structures can tightly trap light at the surface of a metal, which leads to a strongly confined and enhanced local field. ${ }^{[31]}$ As alternative to a microcavity, SPPs provide an easily integrated and ultracompact platform for achieving strong coupling.

DOI: $10.1002 /$ Ipor.201700176 
(a)

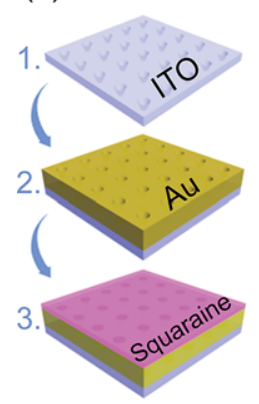

(b)

(c)

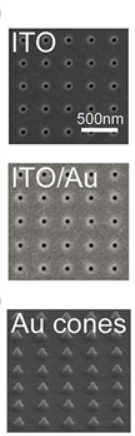

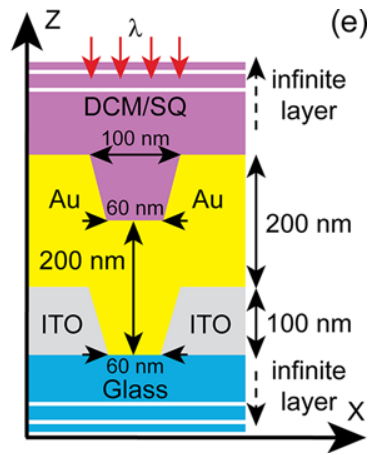

Figure 1. a) Fabrication scheme of the ultrastrong coupled hybrid system: 1. funnel-like shape of the conical pits array fabricated on ITO (100 nm); 2. gold layer deposition (200 nm); 3. spin-coating of SQ dye. b-c) SEM images of the conical pits array on ITO glass without and with Au layer, respectively. d) $52^{\circ}$-tilted SEM images of the template-stripped Au conical pits array. e) Scheme of the structure for the numerical calculation used to determine the reflection profiles.

In ultrastrong coupling regime, the electronic structures of the molecules are significantly modified, with consequences such as ground state energy shift and work function tuning. ${ }^{[32]}$ The strength of the interaction between light and matter is of the same order of magnitude as the frequency of the electronic levels in exciton-like materials or the resonance frequency of the electromagnetic modes. This characteristics implies that the Rotating Wave Approximation (RWA) usually employed for strongly coupled system is no longer applicable to the ultrastrong regime. ${ }^{[1,33]}$ Hence, in such a situation, the photophysics of the hybrid states cannot be predicted from a conventional picture. Furthermore, experiments on the dynamics of ultrastrong coupling systems are still relatively scarce. Therefore, in order to improve the understanding on the kinetics of the hybrid states under ultrastrong coupling, time-resolved spectroscopy experiments should be carried out.

In the present work, ultrastrong exciton-plasmon coupling in squaraine dye filled gold nanoholes array is investigated both from a static and dynamic point of view. A Rabi splitting energy of $860 \mathrm{meV}$, corresponding to $\approx 50 \%$ of the exciton transition energy, is observed in steady-state reflection measurements. Moreover, transient absorption experiments were performed to understand the dynamics of the ultrastrong coupled exciton-plasmon states. It is found that the lower hybrid band is characterized by a shorter lifetime than the uncoupled exciton states, phenomenon which can be attributed to the very strong radiative damping of SPPs modes.

\section{Experimental Section and Simulation}

The fabrication process of the ultrastrong hybrid system is shown in Figure 1a. First, a square-like array of conical pits was milled by means of a focused ion beam in a $100 \mathrm{~nm}$ thick ITO substrate on top of glass. Afterwards, a $200 \mathrm{~nm}$ Au thick film was coated on the ITO substrate by electron beam evaporation. The period of the conical pits array was between 300 and $400 \mathrm{~nm}$, covering a total area of $50 \times 50 \mu \mathrm{m}^{2}$. Scanning electron microscope (SEM) images of a typical conical pits array fabricated in the ITO sub- strate before and after Au deposition are shown in Figure $1 \mathrm{~b}$ and $1 \mathrm{c}$, respectively. In the figures, the top diameter of the conical pits after $\mathrm{Au}$ deposition is equal to $100 \mathrm{~nm}$, while the apex diameter (about $60 \mathrm{~nm}$ ) was measured by template-stripping process, ${ }^{[34]}$ by which the Au conical pits can be easily stripped off to a cured photopolymer film as shown in Figure 1d. The Au-engraved periodic conical pits, which provide the necessary momentum matching condition, act like antennas to couple the incident light into SPPs. In particular, the resonant wavelength can be tuned by acting on parameters such as the period, the incident angle and surrounding material. In this regard, after covering the Au layer with dichloromethane (DCM, refractive index 1.424), the experimental reflection spectra of the structure are shown in Figure 2a. Importantly, all reflection spectra shown in this work have undergone a normalization procedure with respect the reflection from unpatterned gold film. This choice was adopted to mitigate the unphysical reflection increase observed at low wavelengths, in fact due to the limited bandwidth of the adopted lamp and to the scarce reading capabilities below $475 \mathrm{~nm}$. Regardless, we shall stress that this effect is however negligible with respect the observation of strong/ultra-strong coupling regime, as it only affects the hybrid peaks (at low wavelengths) amplitude but not their spectral position.

In Figure $2 \mathrm{a}$ a pronounced reflection peak, associated to the SPPs resonance, is observed for each conical pits array. The SPPs resonance can be tuned from 580 to $650 \mathrm{~nm}$ by modifying the structure period between 300 and $400 \mathrm{~nm}$. Importantly, the SPPs resonance range matches with the broad absorbance spectrum of squaraine (SQ; 2,4-Bis[4-(N,N-diisobutylamino)-2,6dihydroxyphenyl) dye which was chosen as active layer. Indeed, owing to the large oscillator strengths, ${ }^{[28]} \mathrm{SQ}$ dye is considered an ideal candidate for reaching ultrastrong coupling regime. To avoid degradation by oxygen, the sample formed by SQ-DCMplasmonic conical pits array was sealed by a quartz slide under nitrogen environment in glove box with an oxygen concentration lower than $0.1 \mathrm{ppm}$.

In Figure $2 \mathrm{~b}$ are shown the corresponding numerical reflection spectra, calculated by employing the RCWA method. In particular, periodic boundary conditions are assumed in the in-plane (X-Y) directions with the light impinging along $\mathrm{Z}$ direction, as shown in Figure 1e. The gold refractive index is taken from Alabastri et al. ${ }^{[35,36]}$ Importantly, experimental and numerical results show a remarkable matching with respect to the position and the shift of the reflection peak. The discrepancy at low wavelengths is likely due to the choice of infinite DCM layer in the simulations, different from the actual experimental condition.

\section{Results and Discussion}

\subsection{Steady-State Optical Properties of the Ultrastrong Coupled Hybrid States}

In Figure 2 the hybrid system is indeed analyzed through steadystate reflection measurements performed at normal incidence angle using un-polarized light. In particular, the black curve of Figure 2c represents the relative reflection spectrum of a SQ dye layer spin-coated on a flat $\mathrm{Au}$ film taken as reference sample. As mentioned, the reflection spectra are always normalized with 
(a)

ITO/Au/DCM

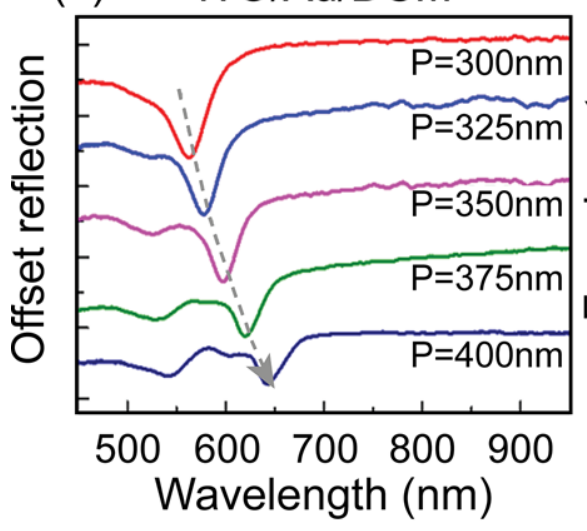

(c)

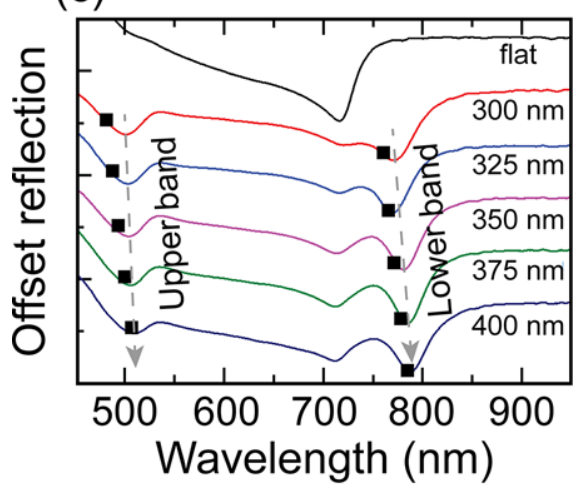

(b)

ITO/Au/DCM

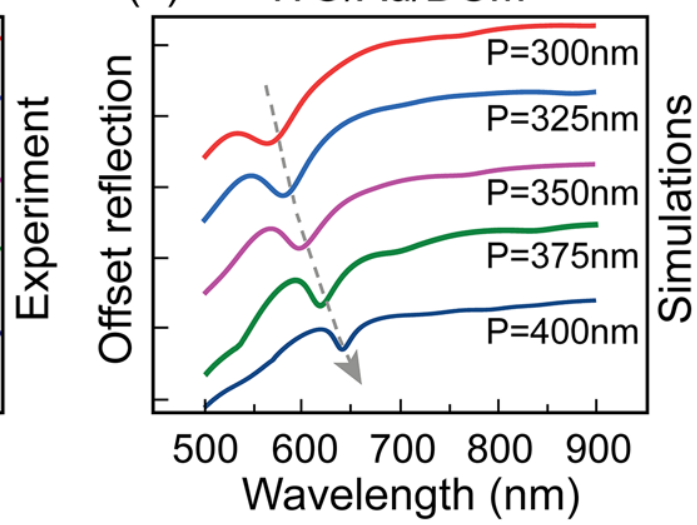

Period (nm)

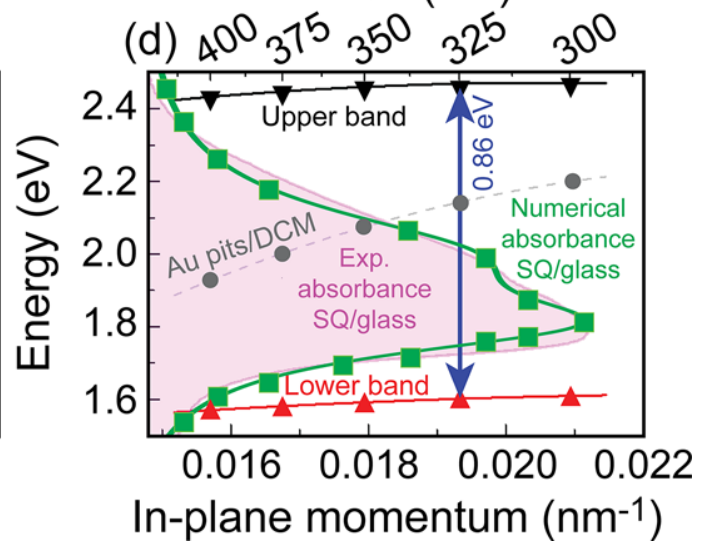

Figure 2. a-b) Respectively experimental and numerical reflection spectra of Au conical pits arrays covered by dichloromethane (DCM). The lattice period is tuned from $300 \mathrm{~nm}$ to $400 \mathrm{~nm}$. c) Reflection spectra from flat gold film (black line) and from Au conical pits arrays (coloured lines) with period from $300 \mathrm{~nm}$ to $400 \mathrm{~nm}$ all covered by a layer of SQ. The gold thickness is $200 \mathrm{~nm}$. The black squares represent the minima of the corresponding numerical simulations. From (a) to (c), the reflection spectra are normalized with pure flat gold film. d) Experimental energy dispersion curves corresponding to the Au conical pits array covered by SQ (black and red triangles, respectively upper and lower hybrid bands) and by DCM (gray dashed line, SPPs resonances as in a). In the background is shown the experimental (pink shadow) and numerical (green/square line) absorbance of SQ dye deposited on top of a glass layer.

respect to unpatterned flat gold film, in order to exclude the influence from the absorption of the film. ${ }^{[37]}$ The role of DCM was here neglected owing to its evaporation occurring after spin-coating. The curve is characterized by a main peak at $715 \mathrm{~nm}$, associated to the absorbance peak of SQ $(\approx 1.8 \mathrm{eV}$, see Figure $2 \mathrm{~d})$, and by a wide line-width from $500 \mathrm{~nm}$ to $750 \mathrm{~nm}$. Afterwards, the relative reflection spectra of the hybrid system formed by SQ dye and different periods of $\mathrm{Au}$ conical pits arrays were measured. In each spectrum (coloured curves) three peaks are clearly visible. Similarly to the balck curve, the peak at $715 \mathrm{~nm}$ is related to the uncoupled SQ dye, indeed it does not show any shift with the tuning of the period. Since the area associated to the Au flat zone increases with the period, it is expected a $715 \mathrm{~nm}$ peak intensity increase moving from 300 to $400 \mathrm{~nm}$ period, as confirmed by the figure. Furthermore, for each period, two extra peaks on both sides of the uncoupled SQ dye reflection peak are shown in the figure, corresponding to the hybrid states formed by the interaction between the SQ dye and SPPs. It is worth noting that the energy difference between the two peaks is larger than both the width of the SPPs refection band (Figure 2a) and SQ dye absorbance spectrum, sug- gesting that the hybrid system reaches indeed strong coupling regime. ${ }^{[1,38]}$ Another important observation is about the possibility that the aforementioned energy difference between the two peaks could be instead explained with Davydov splitting. In fact, based on molecular exciton coupling theory, SQ dye may also lead to band splitting known as Davydov splitting. ${ }^{[39,40]}$ In particular, if present this event should occur also in the reference sample, i.e. in the sample formed by SQ deposited on a flat gold film. Our experimental observations do not however support this scenario, as no splitting is observed in the black curve of Figure 2c. In the same figure are also illustrated the reflection minima associated to the lower and upper bands obtained from the numerical simulations (black square dots), showing a very good match with the experiments. Similarly to Figure 2b, 3D-RCWA method was employed here as well. In this regard, the optical properties of the SQ dye were retrieved by its experimental absorbance, as demonstrated by the comparison between the pink/shadow profile (experimental) and the green/square dots line (numerical) of Figure $2 \mathrm{~d}$. Importantly, this figure also highlights an extra signature of the ultrastrong coupling regime occurring between SPPS 
and the SQ dye, that is the flat-looking anti-crossing behaviour, namely an evident flatness of both the lower and upper bands which prevents from any possible bands crossing ${ }^{[27,29]}$ In particular, the black and red lines correspond to the upper and lower bands of the exciton-plasmon hybrid system, respectively. Both energy levels experience a blue shift with the in-plane momentum $(2 \pi /$ Period). The figure also shows a remarkable Rabi splitting energy around $860 \mathrm{meV}$, defined as the energy difference between the two dispersion branches. In general, when it comes to strongly coupled systems, the Rabi splitting should be measured at the resonant point, namely at the position where the SPPs and the exciton curves come closer (anti-crossing position). In the present case the situation is however different, as the system is in ultra-strong coupling regime, so no anti-crossing behaviour is clearly visible. Instead, both the lower and upper bands show a flat-like form, therefore the Rabi value is roughly constant around $860 \mathrm{meV}$ in all the considered momentum range (i.e. where the $\mathrm{SPPs}$ resonances overlap with the SQ absorbance). This value corresponds to $\approx 50 \%$ of the exciton transition energy which, to our knowledge, is the largest Rabi splitting ever observed involving SPPs. In fact, the corresponding wavelength is $1.44 \mu \mathrm{m}$, close to optical frequencies. Finally, both the absorbance of SQ dye on glass and the SPPs resonances (gray round dots) generated by the $\mathrm{Au}$ pits structure with DCM at different periods are shown. As expected, they fall in the same energy range which is necessary condition for achieving strong/ultrastrong coupling regime.

\subsection{Dynamics of the Ultrastrong Coupled Hybrid States}

Even though the present static approach could demonstrate the achievement of ultrastrong coupling between SPPs and SQ dye, it cannot provide any insight on the nature of the occurring coupling. In particular, no information on the dynamics of the hybrid system under such a giant Rabi splitting can be provided by the static approach. Hence, to gain further insight on the overall system, the dynamics of the hybrid system was analyzed by transient absorption microscopy (TA) ${ }^{[41-43]}$ In particular, in TA experiments we measured the variation of the optical density $\triangle \mathrm{OD}$, here defined as - $\log \left(R_{\text {pump,probe }} / R_{\text {probe }}\right)$, where $R_{\text {pump,probe }}$ is the reflection of the probe laser right after the pump signal has hit the sample, namely the sample reflection upon perturbation induced by the pump laser. Similarly, the $\mathrm{R}_{\text {probe }}$ describes the probe reflectivity in a condition far away from the pump excitation.

A series of TA experiments were performed on hybrid systems with different lattice periods by adopting a $400 \mathrm{~nm}$ pump laser pulses, a value relatively close to the upper band excitation. As reference, TA spectra of SQ dye spin-coated on flat Au film are shown in Figure 3a. The figure illustrates a wide ground state bleaching signal with a main peak at $653 \mathrm{~nm}$ accompanied by a weak shoulder peak extending to $740 \mathrm{~nm}$, feature originating from the SQ dye as it can be infer from Figure 2c (black curve, flat configuration). Furthermore, in the short wavelength region, the spectra show a positive signal which can be attributed to the intrinsic absorption of gold. ${ }^{[44]}$

The TA spectra plotted in Figure 3b-d are characterized by very different features from the spectra of SQ dye on flat Au film shown in Figure 3a. Indeed, while in the short wavelength region the TA spectra are still dominated by the intrinsic absorp- tion of gold, it can be noticed a bleaching peak associated to the lower hybrid energy band at 772, 794 and $800 \mathrm{~nm}$ (depending on the period) which is consistent with the peaks position measured from steady-state reflection measurements. Interestingly, under $400 \mathrm{~nm}$ excitation the bleaching peaks associated to the upper hybrid energy bands do not emerge in the present experiments. This behaviour is due to a rapid vibrational relaxation and to other non-radiative decay processes occurring in the system. ${ }^{[45]}$ In different terms, the absence of the upper bleaching bands is likely originating from the short life time associated to the upper hybrid states, which cannot be resolved by the 100 fs laser setup employed in the present experiment.

Proceeding with the analysis of Figure $3 b-d$, it can be noticed that for the $325 \mathrm{~nm}$ period the bleaching signal from the uncoupled SQ dye molecules is completely absent, while it is present for both the 350 and $375 \mathrm{~nm}$ periods $(\approx$ in the $660 \mathrm{~nm}$ $-690 \mathrm{~nm}$ range). This characteristic suggests the occurring of a full interaction between SQ molecules and SPPs for the $325 \mathrm{~nm}$ period hence providing the ideal condition for ultrastrong coupling regime. On the other hand, for higher periods, less area of the Au film is occupied by the conical pits (hence less SPPs hot spots), leading to an increase of the bleaching signal due to the uncoupled SQ dye molecules. Importantly, these consideration are sustained by the appearing of a strong positive peak on the lower wavelengths side of the lower hybrid band (accompanied by the aforementioned bleaching peak in the range 600 to $690 \mathrm{~nm}$ ) which is attributed to the thermal effect associated to uncoupled SPPs. ${ }^{[46]}$ Indeed, by increasing the period, this peak shows a well expected red shift together with a higher amplitude, the latter behaviour accounting for the hybrid system moving away from the ideal ultrastrong coupling regime condition. In this regard, Figure 4 shows the TA spectra recorded immediately after the initial excitation at $0.5 \mathrm{ps}$ for the Au flat configuration and for the three different periodicities.

Always maintaining a $400 \mathrm{~nm}$ pump laser excitation, Figure 5 compares the normalized bleaching dynamics of SQ dye deposited on flat gold film with the dynamics associated to the lower hybrid bands of the hybrid SQ-SPPs systems with periodicity equal to 325,350 and $375 \mathrm{~nm}$. The plots clearly show the slow decay time associated to the flat configuration when a probe wavelength of $653 \mathrm{~nm}$, corresponding to the bleaching peak, is considered. In particular, the decay appears to be characterized by a multi-exponential behaviour, with a first-half exciton lifetime of about 2 ps, and a longer lifetime component in the nanosecond regime. On the other hand, the lifetimes associated to the hybrid systems, regardless the periodicity, show a faster decay than the flat case. Indeed, a fast decay happening in less than $1 \mathrm{ps}$ is followed by a slower decay tail occurring in hundreds of ps. Interestingly, this result is in contrast with the life time obtained for states in strong coupling regime (where much longer lifetimes are achieved), ${ }^{[4]}$ suggesting that the dynamics in ultrastrong condition can be different from the dynamics for strongly coupled systems. Indeed, the ultrastrong regime leads to excitonSPPs coupling strengths comparable to the frequency of both the exciton oscillator and the SPP mode, which determines the breakdown of the RWA usually employed for describing strong coupling regime. In this regard, in ultrastrong coupling regime the reduction of the lifetime of the hybrid states with respect to the lifetime of bare uncoupled SQ dye can be attributed to the very 
Excitation @ 400 nm
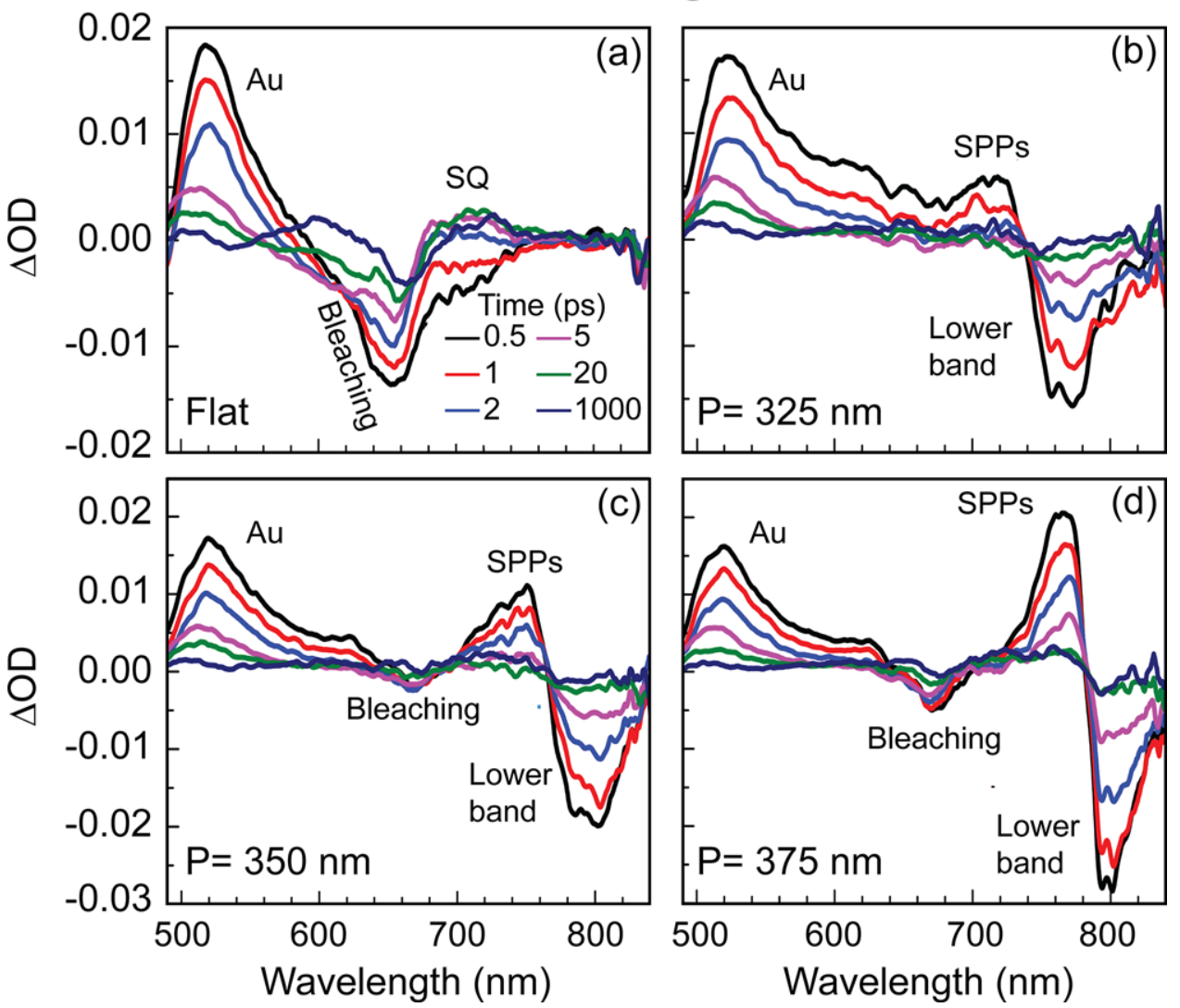

Figure 3. TA spectra of SQ dye on a) a flat gold film and b-d) on different gold conical pits arrays with period equal to 325,350 and $375 \mathrm{~nm}$. As excitation was chosen a $400 \mathrm{~nm}$ laser source. The spectra are recorded at 0.5, 1, 2, 5, 20 and $1000 \mathrm{ps}$. $\triangle \mathrm{OD}$ : optical density variation.

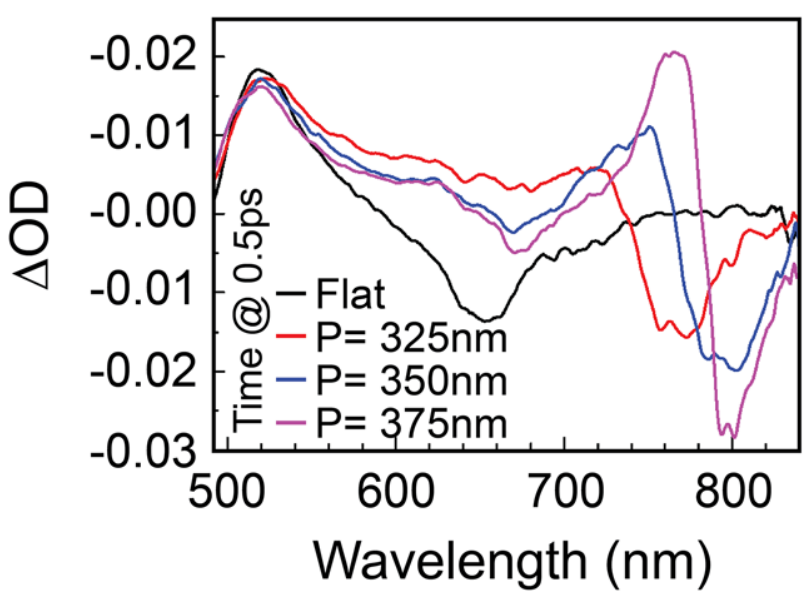

Figure 4. Comparison of the initial TA spectra recorded at $0.5 \mathrm{ps}$ for SQ dye on a flat gold film and on different gold conical pits arrays with periods of 325,350 and $375 \mathrm{~nm}$. The excitation source is taken at $400 \mathrm{~nm}$. $\triangle O D$ : optical density variation.

fast damping of the SPP modes, which is in the range of tens of fs. ${ }^{[4]}$ This can be further understood by carefully analyzing the short lifetime component of the lower hybrid bands for different conical pits periods as shown in the inset of Figure 5. By in-

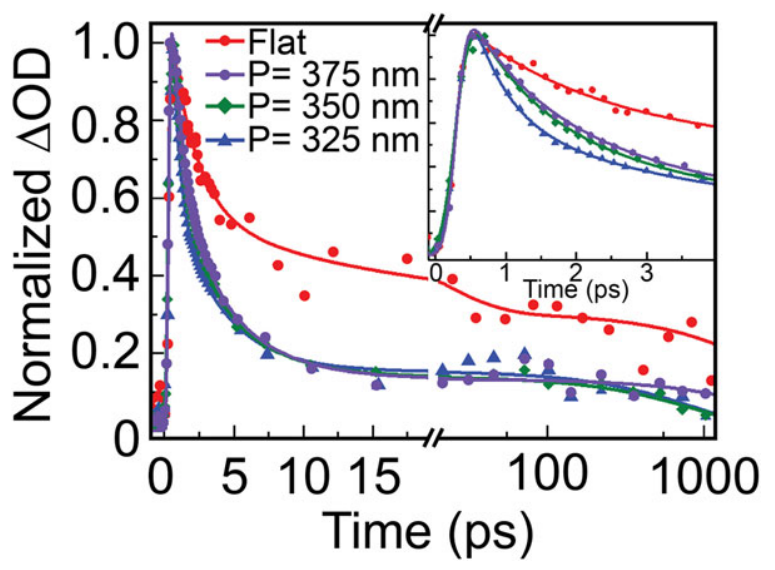

Figure 5. Comparison between the normalized bleaching dynamics at 653 $\mathrm{nm}$ for SQ dye spin-coated on a flat gold film and the dynamics associated to the peaks of the lower bands at different periods. The dynamics in the initial 4 ps are highlighted in the inset. The solid lines represent the fitting results. The excitation source is taken at $400 \mathrm{~nm}$. $\triangle O D$ : optical density variation.

creasing the period of the structure the SPPs contribution tends to decrease owing to the reduction of the conical pits occupied area (the diameter of the conical pits is constant), resulting in longer decay time. In particular, for period equal to $325 \mathrm{~nm}$, the 
lower hybrid state shows the shortest lifetime $(\approx 500 \mathrm{fs})$, which corresponds to a situation where the lower band has the strongest plasmonic character.

\section{Conclusion}

To conclude, we have observed ultrastrong exciton-SPPs coupling in gold conical pits arrays integrated with SQ dye. A giant Rabi splitting of $860 \mathrm{meV}$ was achieved, with the corresponding splitting wavelength close to optical frequencies, which suggests the possibility of using ultrastrong coupling to control the light in this spectral range. Furthermore, the dynamics of hybrid states under ultrastrong coupling regime was studied. It was found that the upper band cannot be determined in the TA spectra, which can be caused by the rapid vibrational relaxation and other nonradiative decay processes. In addition, due to the overwhelming effect due to the very fast damping of the SPP modes, the lower hybrid state shows a lifetime much shorter than the bleaching recovery of SQ dye on a flat gold film, hence providing a substantial difference with the dynamics of strongly coupled systems. Overall, the Rabi splitting energy in our study falls within the telecommunications window, which opens new possibilities to address light managing in the spectral range close to optical frequencies. Importantly, the understanding of the dynamics of ultrastrong coupled hybrid states paves the way towards the realization of devices such as threshold-less lasers and it can improve the knowledge of advanced quantum optical systems. ${ }^{[48-50]}$

\section{Acknowledgements}

The authors would like to recognize the financial support from the $\mathrm{Na}$ tional Science Foundation (awards No. 1102301 and 1254934); from the National Basic Research Program of China (973 Program, Grant No. 2014CB921300), Natural Science Foundation China (NSFC) under Grant no. 21273096 and no. 21473077, the Doctoral Fund Ministry of Education of China under Grant no. 20130061110048 and the 3315 Innovative Teams Program of Ningbo-China.

\section{Conflict of Interest}

The authors declare no conflict of interest.

\section{Keywords}

Rabi splitting, squaraine dyes, surface plasmons, transient absorption measurements, ultrastrong coupling

Received: June 29, 2017

Revised: January 11, 2018

Published online: February 21, 2018

[1] P. Törmä, W. L. Barnes, Rep. Prog. Phys. 2015, 78, 013901.

[2] J. A. Schuller, E. S. Barnard, W. Cai, Y. C. Jun, J. S. White, M. L. Brongersma, Nat. Mater. 2010, 9, 193.

[3] H. Wang, H.-Y. Wang, B.-R. Gao, Y. Jiang, Z.-Y. Yang, Y.-W. Hao, Q.-D. Chen, X.-B. Du, H.-B. Sun, Appl. Phys. Lett. 2011, 98, 251501.

[4] Y. Jiang, H.-Y. Wang, H. Wang, B.-R. Gao, Y.-w. Hao, Y. Jin, Q.-D. Chen, H.-B. Sun, J. Phys. Chem. C 2011, 115, 12636.

[5] Y.-W. Hao, H.-Y. Wang, Y. Jiang, Q.-D. Chen, K. Ueno, W.-Q. Wang, H. Misawa, H.-B. Sun, Angew. Chem. 2011, 123, 7970.
[6] Y.-Y. Lai, Y.-P. Lan, T.-C. Lu, Light Sci. Appl. 2013, 2, e76.

[7] D. G. Lidzey, D. D. C. Bradley, M. S. Skolnick, T. Virgili, S. Walker, D. M. Whittaker, Nature 1998, 395, 53.

[8] R. Ameling, H. Giessen, Laser Photo. Rev. 2013, 7, 141.

[9] R. Chikkaraddy, B. de Nijs, F. Benz, S. J. Barrow, O. A. Scherman, E. Rosta, A. Demetriadou, P. Fox, O. Hess, J. J. Baumberg, Nature 2016, 535, 127.

[10] K. Santhosh, O. Bitton, L. Chuntonov, G. Haran, Nat. Commun. 2016, 7, 11823.

[11] M. A. Noginov, G. Zhu, A. M. Belgrave, R. Bakker, V. M. Shalaev, E. E. Narimanov, S. Stout, E. Herz, T. Suteewong, U. Wiesner, Nature 2009, 460, 1110.

[12] B. Zhang, Z. Wang, S. Brodbeck, C. Schneider, M. Kamp, S. Hofling, H. Deng, Light Sci. Appl. 2014, 3, e135.

[13] R.-M. Ma, R. F. Oulton, V. J. Sorger, X. Zhang, Laser Photo. Rev. 2013, $7,1$.

[14] T. K. Hakala, H. T. Rekola, A. I. Väkeväinen, J. P. Martikainen, M. Nečada, A. J. Moilanen, P. Törmä, Nat. Commun. 2017, 8, 13687.

[15] J. D. Plumhof, T. Stöferle, L. Mai, U. Scherf, R. F. Mahrt, Nat. Mater. 2014, 13, 247.

[16] S. Balci, C. Kocabas, B. Küçüköz, A. Karatay, E. Akhüseyin, H. Gul Yaglioglu, A. Elmali, Appl. Phys. Lett. 2014, 105, 051105.

[17] B. G. DeLacy, O. D. Miller, C. W. Hsu, Z. Zander, S. Lacey, R. Yagloski, A. W. Fountain, E. Valdes, E. Anquillare, M. Soljačić, S. G. Johnson, J. D. Joannopoulos, Nano Lett. 2015, 15, 2588.

[18] A. E. Schlather, N. Large, A. S. Urban, P. Nordlander, N. J. Halas, Nano Lett. 2013, 13, 3281.

[19] H. Wang, A. Toma, H.-Y. Wang, A. Bozzola, E. Miele, A. Haddadpour, G. Veronis, F. De Angelis, L. Wang, Q.-D. Chen, H.-L. Xu, H.-B. Sun, R. P. Zaccaria, Nanoscale 2016, 8, 13445.

[20] H. Wang, H.-Y. Wang, A. Bozzola, A. Toma, S. Panaro, W. Raja, A. Alabastri, L. Wang, Q.-D. Chen, H.-L. Xu, F. De Angelis, H.-B. Sun, R. P. Zaccaria, Adv. Funct. Mater. 2016, 26, 6198.

[21] F. Valmorra, M. Bröll, S. Schwaiger, N. Welzel, D. Heitmann, S. Mendach, Appl. Phys. Lett. 2011, 99, 051110.

[22] T. K. Hakala, J. J. Toppari, A. Kuzyk, M. Pettersson, H. Tikkanen, H. Kunttu, P. Törmä, Phys. Rev. Lett. 2009, 103, 053602.

[23] H. Wang, H.-Y. Wang, L. Wang, Q.-D. Chen, H.-L. Xu, A. Carrara, R. Proietti Zaccaria, H.-B. Sun, A. Toma, Adv. Opt. Mater. 2017, 5, 1600857.

[24] D. E. Gómez, S. S. Lo, T. J. Davis, G. V. Hartland, J. Phys. Chem. B 2012, 117, 4340

[25] D. E. Gómez, K. C. Vernon, P. Mulvaney, T. J. Davis, Nano Lett. 2010 , 10, 274.

[26] H. Wang, H.-Y. Wang, A. Toma, T.-a. Yano, Q.-D. Chen, H.-L. Xu, H.-B. Sun, R. Proietti Zaccaria, J. Phys. Chem. Lett. 2016, 7, 4648.

[27] T. Schwartz, J. A. Hutchison, C. Genet, T. W. Ebbesen, Phys. Rev. Lett. 2011, 106, 196405.

[28] S. Gambino, M. Mazzeo, A. Genco, O. Di Stefano, S. Savasta, S. Patanè, D. Ballarini, F. Mangione, G. Lerario, D. Sanvitto, G. Gigli, ACS Photonics 2014, 1, 1042.

[29] S. Kéna-Cohen, S. A. Maier, D. D. C. Bradley, Adv. Opt. Mater. 2013, $1,827$.

[30] A. Cacciola, O. Di Stefano, R. Stassi, R. Saija, S. Savasta, ACS Nano 2014, 8, 11483.

[31] W. L. Barnes, A. Dereux, T. W. Ebbesen, Nature 2003, 424, 824.

[32] J. A. Hutchison, A. Liscio, T. Schwartz, A. Canaguier-Durand, C. Genet, V. Palermo, P. Samorì, T. W. Ebbesen, Adv. Mater. 2013, 25, 2481.

[33] Y. Wu, X. Yang, Phys. Rev. Lett. 2007, 98, 013601.

[34] Y.-F. Liu, J. Feng, H.-F. Cui, Y.-F. Zhang, D. Yin, Y.-G. Bi, J.-F. Song, Q.-D. Chen, H.-B. Sun, Nanoscale 2013, 5, 10811.

[35] A. Alabastri, A. Toma, M. Malerba, F. De Angelis, R. Proietti Zaccaria, ACS Photonics 2015, 2, 115. 
[36] A. Alabastri, S. Tuccio, A. Giugni, A. Toma, C. Liberale, G. Das, F. Angelis, E. Fabrizio, R. Zaccaria, Materials 2013, 6, 4879.

[37] Z. Wang, Z. Dong, Y. Gu, Y.-H. Chang, L. Zhang, L.-J. Li, W. Zhao, G Eda, W. Zhang, G. Grinblat, S. A. Maier, J. K. W. Yang, C.-W. Qiu, A. T. S. Wee, Nat. Commun. 2016, 7, 11283.

[38] J. Dintinger, S. Klein, F. Bustos, W. L. Barnes, T. W. Ebbesen, Phys. Rev. B 2005, 71, 035424

[39] H.-H. Fang, J. Yang, J. Feng, T. Yamao, S. Hotta, H.-B. Sun, Laser Photo. Rev. 2014, 8, 687.

[40] A. S. Davydov, Soviet Physics Uspekhi 1964, 7, 145.

[41] B.-R. Gao, H.-Y. Wang, Z.-Y. Yang, H. Wang, L. Wang, Y. Jiang, Y.-W. Hao, Q.-D. Chen, Y.-P. Li, Y.-G. Ma, H.-B. Sun, J. Phys. Chem. C 2011, $115,16150$.

[42] L. Wang, Q. Li, H.-Y. Wang, J.-C. Huang, R. Zhang, Q.-D. Chen, H.-L. Xu, W. Han, Z.-Z. Shao, H.-B. Sun, Light Sci. Appl. 2015, 4, e245.
[43] D. y. Davydova, A. de la Cadena, D. Akimov, B. Dietzek, Laser Photo. Rev. 2016, 10, 62

[44] N. J. Halas, S. Lal, W.-S. Chang, S. Link, P. Nordlander, Chem. Rev. 2011, 111, 3913.

[45] T. Schwartz, J. A. Hutchison, J. Léonard, C. Genet, S. Haacke, T. W. Ebbesen, Chemphyschem 2013, 14, 125.

[46] G. V. Hartland, Chem. Rev. 2011, 111, 3858.

[47] M. Pelton, J. Aizpurua, G. Bryant, Laser Photo. Rev. 2008, 2, 136.

[48] R. F. Oulton, V. J. Sorger, T. Zentgraf, R.-M. Ma, C. Gladden, L. Dai, G. Bartal, X. Zhang, Nature 2009, 461, 629.

[49] R. Bose, T. Cai, K. R. Choudhury, G. S. Solomon, E. Waks, Nat. Photonics 2014, 8, 858.

[50] J.-X. Li, Y. Xu, Q.-F. Dai, S. Lan, S.-L. Tie, Laser Photo. Rev. 2016, 10, 826 